\title{
FTY720 protects neuronal cells from damage induced by human prion protein by inactivating the JNK pathway
}

\author{
MYUNG-HEE MOON, JAE-KYO JEONG, YOU-JIN LEE and SANG-YOUEL PARK \\ Biosafety Research Institute, College of Veterinary Medicine, \\ Chonbuk National University, Jeonju, Jeonbuk 561-756, Republic of Korea
}

Received July 20, 2013; Accepted October 8, 2013

DOI: $10.3892 /$ ijmm.2013.1528

\begin{abstract}
Prion diseases affect the central nervous system (CNS) in humans and animals, and are associated with the conversion of the cellular prion protein $\left(\mathrm{PrP}^{\mathrm{C}}\right)$ to the misfolded isoform $\left(\mathrm{PrP}^{\mathrm{Sc}}\right)$. FTY720, an immune modulator and synthetic analogue of sphingosine-1-phosphate (S1P), activates S1P receptors and has been shown to be effective in experimental models of transplantation and autoimmunity, including multiple sclerosis. Whereas the immune modulatory functions of FTY720 have been extensively investigated, the other functions of FTY720 are not yet well understood. In this study, we investigated the effects of FTY720 phosphate (FTY720-p) on prion protein-mediated neuronal cell death, as well as its effects on intracellular apoptotic pathways. Treatment with FTY720-p protected neuronal cells from synthetic human prion protein peptide [PrP (106-126)]-mediated damage and prevented mitochondrial dysfunction by inhibiting the activation of c-jun N-terminal kinase. Moreover, FTY720-p prevented the PrP (106-126)-induced reduction in mitochondrial potential, the translocation of Bax to the mitochondria and the release of cytochrome $c$. To the best of our knowledge, this study is the first to demonstrate the effects of FTY720 on prion protein-mediated neurotoxicity and to suggest that FTY720 has therapeutic potential in prion diseases.
\end{abstract}

\section{Introduction}

Transmissible spongiform encephalopathies (TSEs) or prion diseases are fatal neurodegenerative disorders of the central nervous system (CNS) in humans [Kuru, Creutzfeldt-Jakob disease (CJD), fatal familial insomnia (FFI) and Gerstmann

Correspondence to: Professor Sang-Youel Park, Biosafety Research Institute, College of Veterinary Medicine, Chonbuk National University, 664-14 Duckjin-dong, Jeonju, Jeonbuk 561-756, Republic of Korea

E-mail: sypark@chonbuk.ac.kr

Key words: human prion protein, FTY720, c-jun N-terminal kinase, mitochondrial transmembrane potential, Bax translocation
Straussler Sheinker syndrome (GSS)] and animals (bovine spongiform encephalopathy in cattle and scrapie in sheep or goats) $(1,2)$. TSEs are characterized by the spongiform degeneration of the CNS, astrogliosis and the deposition of amyloid fibrils in the brain $(1,3)$. In prion diseases, the normal cellular form of the prion protein $\left(\mathrm{PrP}^{\mathrm{C}}\right)$ undergoes a conformational conversion to the $\beta$-sheet-rich scrapie isoform $\left(\mathrm{PrP}^{\mathrm{Sc}}\right)$, which is partially resistant to protease digestion $(4,5)$. The conformational change into $\mathrm{PrP}^{\mathrm{Sc}}$ occurs through unknown molecular mechanisms.

One of the mechanisms of neuronal cell death in prion diseases is apoptosis, as apoptotic neurons have been observed in the brains of scrapie-infected sheep and patients with CJD $(6,7)$. The synthetic human prion protein peptide [PrP (106-126)] maintains many characteristics of PrP ${ }^{\mathrm{Sc}}$. These include the ability to form amyloid fibrils and induce apoptosis in primary rat hippocampal cultures (8), primary mouse cerebellar cultures (9), GH3 rat clonal pituitary cells (10), as well as in mouse retinae (11). Cortical neuron cells treated with the PrP fragment (106-126) have been shown to become neurotoxic, develop dysfunctional mitochondria and display increased prion-mediated neurotoxicity associated with the induction of Bax translocation to the mitochondria $(12,13)$. These characteristics of the 106-126 sequence of the prion protein render it a useful, in vitro model for the study of the pathogenesis of prion diseases (10).

FTY720 \{2-amino-2-[2-(4-n-octylphenyl)ethyl]1,3-propanediol hydrochloride is synthetically derived from myriocin (ISP-1), a metabolite isolated from the ascomycete Isaria sinclarii (14). The pharmacokinetics of FTY720 have been characterized extensively, and have shown clinical efficacy in phase 3 clinical trials involving patients with multiple sclerosis (MS) (15). It is a prodrug that is phosphorylated by type 2 sphingosine kinase to form FTY720-phosphate (FTY720-p) (16). In addition to its role in T-cell sequestration, the lipophilic nature of FTY720 allows it to readily cross the blood-brain barrier and exert a number of direct effects on the CNS $(17,18)$. These include the regulation of myelination and microglial activation following injury, proliferation and the migration of neural precursor cells toward injury sites, as well as the potentiation of growth-factor regulated neuronal differentiation and survival (18-22). FTY720 is capable of increasing the production of brain-derived neurotrophic factor (BDNF), an endogenous neuroprotectant, in neuronal 
cultures $(17,23)$. Thus, some physiological effects of FTY720 are related to its neuroprotective effects. However, to the best of our knowledge, the effects of FTY720 on neurodegenerative diseases, including prion-mediated neurotoxicity have not yet been reported.

The present study focused on the effects of FTY720 on PrP (106-126)-induced apoptosis and whether FTY720 can be used to prevent mitochondrial dysfunction in prion diseases. We demonstrate that the treatment of neuronal cells with FTY720 inhibits PrP (106-126)-induced neurotoxicity and mitochondrial dysfunction by blocking the phosphorylation of c-jun N-terminal kinase (JNK). This suggests that FTY720 has therapeutic potential in mitochondrial dysfunction-related neurodegenerative disorders, including prion diseases.

\section{Materials and methods}

Cell culture. Human neuroblastoma cells (SH-SY5Y) were obtained from the American Type Culture collection (ATCC; Rockville, MD, USA). The cells were cultured in minimum essential medium (MEM) (HyClone Laboratories, Logan, UT, USA) that contained $10 \%$ fetal bovine serum (InvitrogenGibco, Grand Island, NY, USA) and gentamycin $(0.1 \mathrm{mg} / \mathrm{ml})$, in a humidified incubator maintained at $37^{\circ} \mathrm{C}$ and $5 \% \mathrm{CO}_{2}$.

$\operatorname{PrP}$ (106-126) treatment. Synthetic $\operatorname{PrP}$ (106-126) (sequence, Lys-Thr-Asn-Met-Lys-His-Met-Ala-Gly-Ala-Ala-Ala-Ala-GlyAla-Val-Val-Gly-Gly-Leu-Gly) peptides were synthesized from Peptron (Seoul, Korea). The peptides were dissolved in sterile DMSO at a concentration of $12.5 \mathrm{mM}$ and stored at $-80^{\circ} \mathrm{C}$.

Lactate dehydrogenase ( $L D H)$ assay. Cytotoxicity was assessed by LDH assay in supernatant medium, using an LDH Cytotoxicity Detection kit (Takara Bio, Inc., Tokyo, Japan) according to the manufacturer's instructions. LDH activity was determined by measuring the absorbance at $490 \mathrm{~nm}$ using a microplate reader (Spectra Max M2; Molecular Devices, LLC, Sunnyvale, CA, USA).

Annexin $V$ assay. Apoptosis was assessed by Annexin V assay in the detached cells using an Annexin V assay kit (Santa Cruz Biotechnology, Inc., Santa Cruz, CA, USA) according to the manufacturer's instructions. The number of Annexin V-positive cells was determined by measuring the fluorescence at excitation $488 \mathrm{~nm}$ and emission 525/30 using Guava easyCyte HT System (Millipore, Billerica, MA, USA).

Western blot analyses. The SH-SY5Y cells were lysed in lysis buffer (25 mM HEPES; pH 7.4, 100 mM NaCl, 1 mM EDTA, $5 \mathrm{mM} \mathrm{MgCl} 2,0.1 \mathrm{mM}$ DTT and protease inhibitor mixture). Proteins were electrophoretically resolved on a $10-15 \%$ sodium dodecyl sulfate (SDS) gel and immunoblotting was performed as previously described (5). Equal amounts of lysate protein were resolved on a 10-15\% SDS-polyacrylamide gel and electrophoretically transferred onto a nitrocellulose membrane. Immunoreactivity was detected through sequential incubation with horseradish peroxidase-conjugated secondary antibodies and ECL reagents. The antibodies used for immunoblotting were caspase-3, phospho-JNK, Bax, cytochrome $c$ (both from Cell Signaling Technology, Inc., Beverly, MA, USA) and $\beta$-actin (Sigma-Aldrich, St. Louis, MO, USA). Images were examined using the Fusion-FX7 imaging system (Vilber Lourmat, Marne-la-Vallée, France).

Immunofluorescence staining. The SH-SY5Y cells cultured on glass slides were fixed with cold acetone, blocked with 5\% fetal bovine serum in TBST and incubated with mouse phosphorylated JNK (p-JNK; Cell Signaling Technology) and rabbit active caspase-3 antibodies (R\&D Systems, Minneapolis, MN, USA) overnight at $4^{\circ} \mathrm{C}$. After being washed with TBST, the cells were incubated with goat anti-mouse IgG conjugated with Alexa Fluor ${ }^{\circledR} 488$ (green) and goat anti-rabbit IgG conjugated with Alexa Fluor ${ }^{\circledR} 546$ (red). The cells were washed with TBST, mounted with fluorescence mounting medium and observed under a fluorescence microscope (Nikon Eclipse 80i; Nikon Corp., Tokyo, Japan). Images were captured using a Nikon digital camera, and processed with the appropriate software (Diagnostic Instruments, Victoria Park, Australia).

Cellular fractionation. The SH-SY5Y cells were resuspended in mitochondrial buffer ( $210 \mathrm{~mm}$ sucrose, $70 \mathrm{~mm}$ mannitol, $1 \mathrm{~mm}$ EDTA, $10 \mathrm{~mm}$ HEPES), broken by a 26-gauge needle, and centrifuged at $700 \mathrm{x}$ g for $10 \mathrm{~min}$. The post-nuclear supernatant was centrifuged at $10,000 \mathrm{xg}$ for $30 \mathrm{~min}$. The pellet was used as the mitochondrial fraction and the supernatant was used as the cytosolic fraction. Total proteins were obtained and subjected to western blot analysis.

Mitochondrial transmembrane potential (MTP) assay. The changes in MTP were evaluated using a cationic fluorescent indicator (JC-1; Molecular Probes, Eugene, OR, USA), which aggregates in intact mitochondria (red fluorescence) indicating high or normal MTP and low MTP when it remains in a monomeric form in the cytoplasm (green fluorescence). The SH-SY5Y cells were incubated in MEM containing $10 \mathrm{ml}$ JC-1 at $37^{\circ} \mathrm{C}$ for $15 \mathrm{~min}$, washed with PBS and subsequently transferred to a clear 96-well plate. JC-1 aggregate fluorescence emission was measured at $583 \mathrm{~nm}$, with an excitation wavelength of $526 \mathrm{~nm}$. JC-1 monomer fluorescence intensity was also measured with both excitation and emission wavelengths at 525 and $530 \mathrm{~nm}$, respectively using a microplate reader (SpectraMax M2; Molecular Devices) or a Guava easyCyte HT System. The SH-SY5Y cells were cultured on cover slips in a 24-well plate, incubated in MEM containing $10 \mathrm{ml} \mathrm{JC}-1$ at $37^{\circ} \mathrm{C}$ for $15 \mathrm{~min}$ and then washed with PBS. Finally, the cells were mounted with DakoCytomation fluorescent mounting medium and visualized under a fluorescence microscope.

Statistical analysis. All data are expressed as the the means \pm standard deviation (SD), and the data were compared using the Student's t-test, as well as ANOVA and Duncan multiple range tests with the SAS statistical package. In the figures, mean values denoted by a common alphabetical symbol do not differ significantly. Bars labeled with different letters indicate significant differences among each group of bars according to Duncan's test $(\mathrm{p}<0.05)$. 
(A)
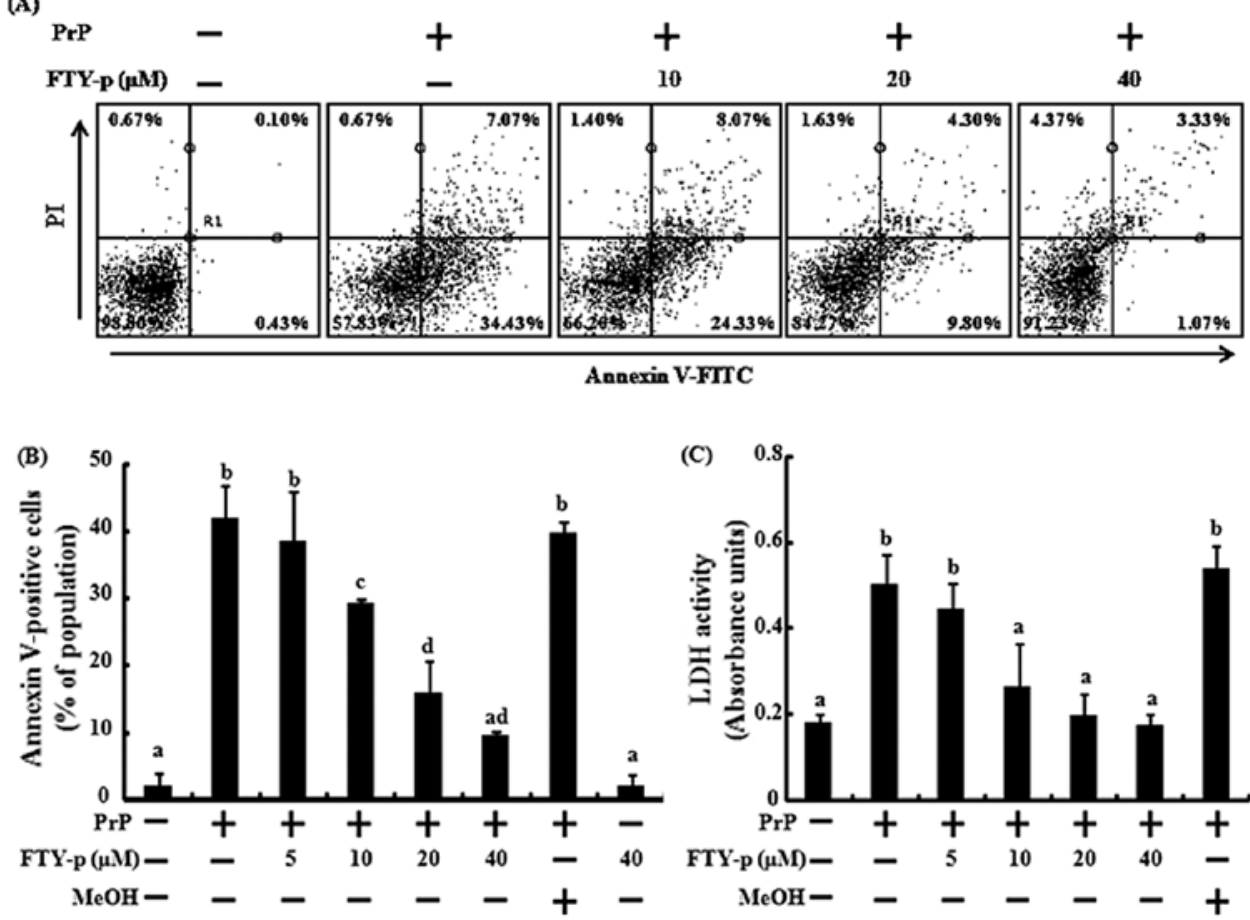

Figure 1. Administration of FTY720 phosphate (FTY-p) protects neuronal cells from PrP (106-126)-induced damage. (A) SH-SY5Y neuronal cells were pre-treated for $1 \mathrm{~h}$ with various doses of FTY-p and then exposed to $50 \mu \mathrm{M}$ PrP (106-126) for $24 \mathrm{~h}$. Cell viability was measured by Annexin V assay. (B) Bar graph indicates the averages of Annexin V-positive cells. (C) Cells were treated with the indicated concentrations of PrP (106-126) for 23 h following exposure to FTY-p for $1 \mathrm{~h}$. The release of lactate dehydrogenase (LDH) into the cell culture supernatant from damaged cells was measured. Data were analyzed using analysis of variance (ANOVA) and the Duncan multiple range test $(\mathrm{p}<0.05)$. Bars indicate the means \pm SD $(n=3)$. The experiments were repeatedly performed to confirm the results. Data were analyzed using analysis of variance (ANOVA) and the Duncan multiple range test (p<0.05). Mean values denoted by a common alphabetical symbol do not differ significantly. Bars labeled with different letters indicate significant differences among each group of bars according to Duncan's test $(\mathrm{p}<0.05)$.

\section{Results}

FTY720-p inhibits PrP (106-126)-induced neuronal cell death. We used $\operatorname{PrP}(106-126)$ to examine $\mathrm{PrP}^{\mathrm{Sc}}$ pathogenesis through the triggering of cell death signals and evaluated the effects of FTY720 on PrP (106-126)-induced neuronal cell death. FTY720 is phosphorylated in vivo by sphingosine kinase 2 to become the active drug metabolite, (S)-FTY720-p, and only the (S)-phosphorylated form of FTY720 is capable of activating sphingosine-1-phosphate (S1P) receptors in vitro $(24,25)$. Therefore, we examined the effects of FTY720-p on PrP (106-126)-induced neurotoxicity in the SH-SY5Y cells. To examine the neuroprotective effects of FTT720-p, we examined the effects of FTY720-p on PrP (106-126)-mediated neurotoxicity in SH-SY5Y cells by Annexin V assay. The SH-SY5Y cells were pre-treated with various doses of FTY720-p $(5,10,20$ and $40 \mu \mathrm{M})$ prior to exposure to $50 \mu \mathrm{M} \operatorname{PrP}(106-126)$ for $24 \mathrm{~h}$. The cells were responsive to $\operatorname{PrP}(106-126)$ treatment $(41.5 \%$ increase in Annexin V-positive cells), and FTY720-p had no effect on cell viability. However, treatment with FTY720-p inhibited PrP (106-126)-induced neuronal cell death. The effects of FTY720-p were detected at $10 \mu \mathrm{M}$ and were maximal at $40 \mu \mathrm{M}$ (Fig. 1A and B). The protective effects of FTY720-p against PrP (106-126)-mediated toxicity were further confirmed by the determination of LDH release as a marker of cytotoxicity. Assays of LDH activity in the cell culture supernatants demonstrated that FTY720-p significantly inhibited PrP (106-126)-induced cytotoxicity in SH-SY5Y cells (Fig. 1C). Consistent with these results, immunoblot analysis of activated caspase-3 revealed that treatment with FTY720-p markedly inhibited PrP (106-126)-induced apoptosis (Fig. 3A and $\mathrm{D})$. These results indicated that prion-induced neuronal cell death was inhibited by FTY720-p.

FTY720-p protects neuronal cells from PrP (106-126)-mediated mitochondrial dysfunction. We then assessed whether the protective effects of FTY720-p on PrP (106-126)-mediated neurotoxicity were related to the prevention of mitochondrial dysfunction. The SH-SY5Y cells were pre-incubated with 1 or $10 \mu \mathrm{M}$ FTY720-p for $1 \mathrm{~h}$ and then exposed to $50 \mu \mathrm{M}$ PrP (106-126). The PrP (106-126)-treated cells exhibited increased JC-1 monomers (79.74\%), indicating low MTP values, while treatment with FTY720-p reduced the number of PrP (106-126)-induced JC-1 monomers (58.48\%), indicating an increase in MTP values (Fig. 2A). The fluorescence microscopy images (Fig. 2B) confirmed the results, depicting cells with green fluorescence (JC-1 monomer form) following exposure to $\operatorname{PrP}$ (106-126), thus indicating lower MTP values, while the control cells and FTY720-p treated cells exhibited red fluorescence (JC-1 aggregates form), indicating high MTP values. Given that Bax proteins act downstream in the mitochondrial apoptotic pathway, we examined the effects of FTY720-p on PrP (106-126)-induced Bax translocation 

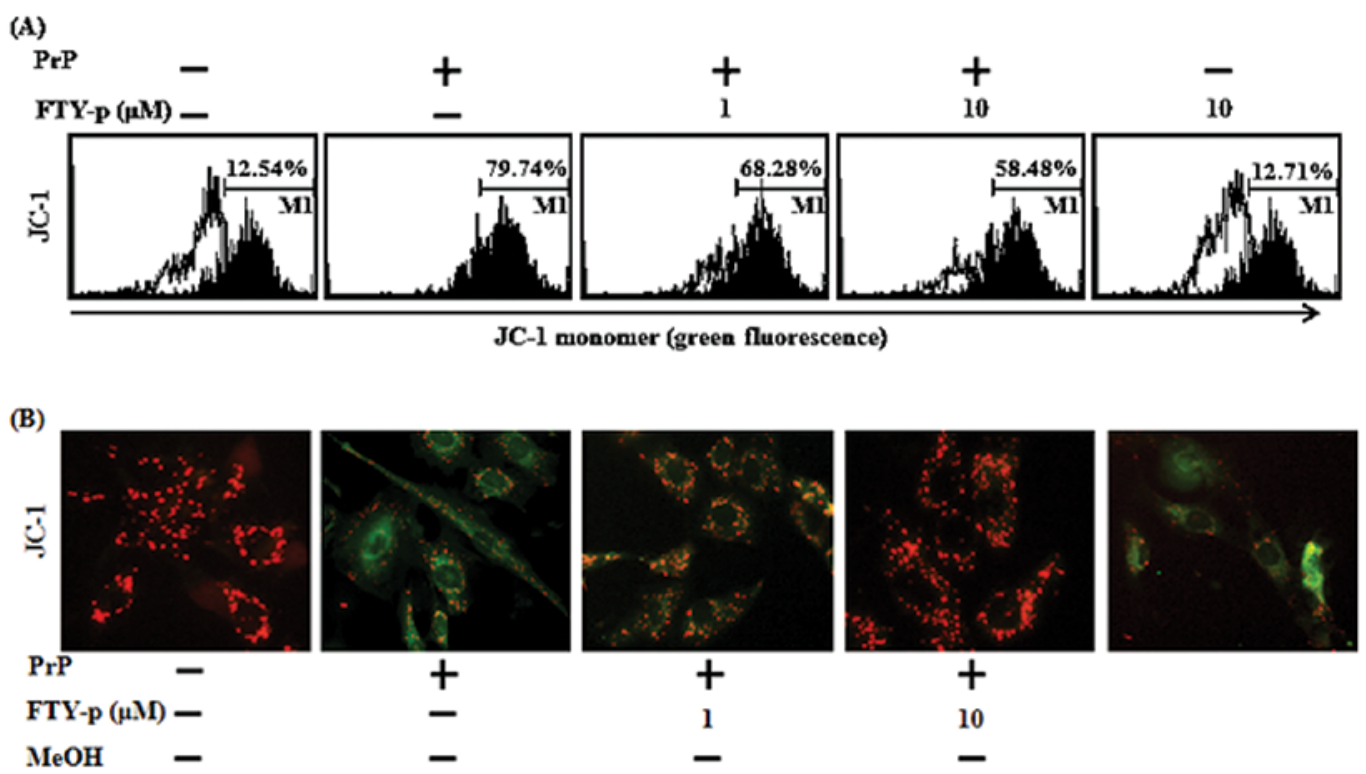

(C)

Bax

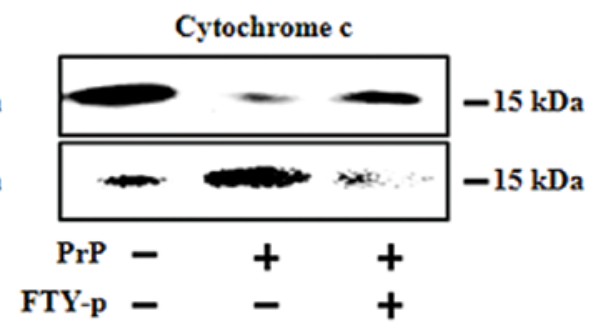

Figure 2. FTY720 phosphate (FTY-p) protects neuronal cells from PrP (106-126)-induced mitochondrial dysfunction. (A) SH-SY5Y cells were pre-treated with $10 \mu \mathrm{M}$ of FTY-p $(1 \mathrm{~h})$ and then exposed to $50 \mu \mathrm{M} \operatorname{PrP}(106-126)$ for $24 \mathrm{~h}$. JC-1 mono form (green) was measured in the treated cells by flow cytometry. M1 represents the population of JC-1 monomeric cells. (B) Representative images of JC-1 aggregate formation in the treated cells, as described in (A). JC-1 aggregate forms (red) and mono forms (green) were measured in the treated cells by confocal microscopy analysis; scale bar, $50 \mu \mathrm{m}$. (C) Cells were homogenized in a mitochondrial buffer, as described in Materials and methods. The separation of cytosol (Cyto) and mitochondrial (Mito) extracts was analyzed by western blot analysis using antibodies against cytochrome $c$ and Bax protein.

and the release of cytochrome $c$. Exposure to $\operatorname{PrP}$ (106-126) induced the translocation of Bax to the mitochondria and the release of cytochrome $c$ release into the cytosol of SH-SY5Y cells. The PrP (106-126)-induced Bax translocation and release of cytochrome $c$ were inhibited by treatment with FTY720-p (Fig. 2C). Overall these results are consistent with the idea that FTY720-p blocks PrP (106-126)-induced apoptosis by preventing mitochondrial dysfunction.

Administration of FTY720-p inhibits PrP (106-126)-induced neurotoxicity by regulating the activation of JNK proteins. $\mathrm{JNK}$ promotes the translocation of Bax to the mitochondria and the release of mitochondrial cytochrome $c$, leading to cell apoptosis $(26,27)$. To gain insight into the molecular mechanisms responsible for the observed biological effects of FTY720, we examined the ability of FTY720-p to inactivate this protein kinase. The SH-SY5Y cells were pre-incubated with various concentrations of FTY720-p for $1 \mathrm{~h}$ and then exposed to PrP (106-126) (Fig. 3). The PrP (106-126)-treated cells displayed increased protein levels of p-JNK (Fig. 3A). By contrast, treatment with FTY720-p decreased p-JNK protein levels in the SH-SY5Y cells treated with $\operatorname{PrP}(106-126)$ (Fig. 3A-C) in a dose-dependent manner. These results indicate that treatment with FTY720-p prevents prion peptide-induced apoptosis by regulating JNK activation.

To determine whether FTY720 functions by inactivating JNK to inhibit PrP (106-126)-induced apoptosis, the apoptosis of the SH-SY5Y cells was induced by exposure to $\operatorname{PrP}$ (106-126); the cells were either pre-treated with $10 \mu \mathrm{M}$ of FTY720-p or $2 \mu \mathrm{M}$ SP600125 (a JNK inhibitor). Pre-treatment with SP600125 and FTY720-p was sufficient to block the phosphorylation of JNK induced by PrP (106-126) (Fig. 4D). When SP600125 was added to the cell cultures, it prevented the increase of Annexin $\mathrm{V}$ binding to membranes due to PrP (106-126) (Fig. 4A and B). The effects of SP600125 were detected at $2 \mu \mathrm{M}$. Treatment with $2 \mu \mathrm{M}$ SP600125 decreased the levels of the active form of caspase-3 to levels similar to those observed with FTY720-p treatment (Fig. 4D).

We then we wished to determine the effects of FTY720induced JNK inactivation on PrP (106-126)-induced mitochondrial dysfunction. The SH-SY5Y cells were pretreated with FTY720-p or SP600125 and then exposed to PrP (106-126). Treatment with SP600125 blocked the low MTP values induced by PrP (106-126), similar to FTY720-p treatment (Fig 4C). Treatment of the cells with SP600125 prevented the PrP (106-126)-induced translocation of Bax and 
(A)

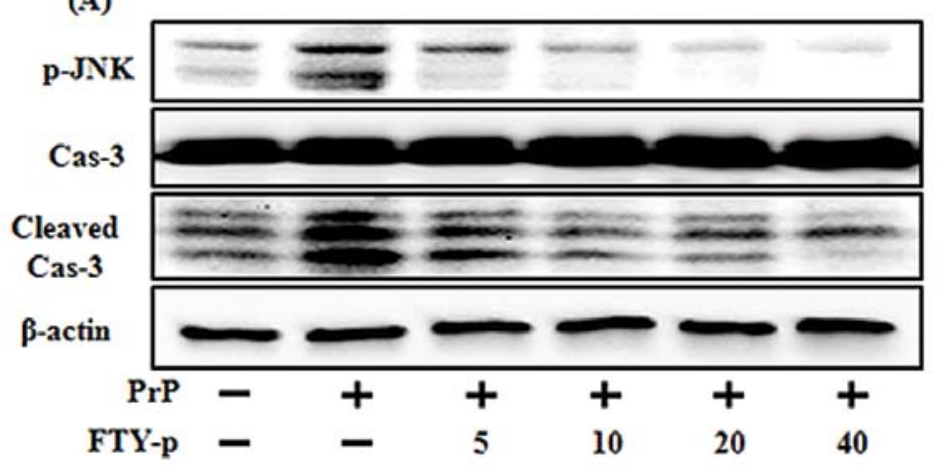

(B)

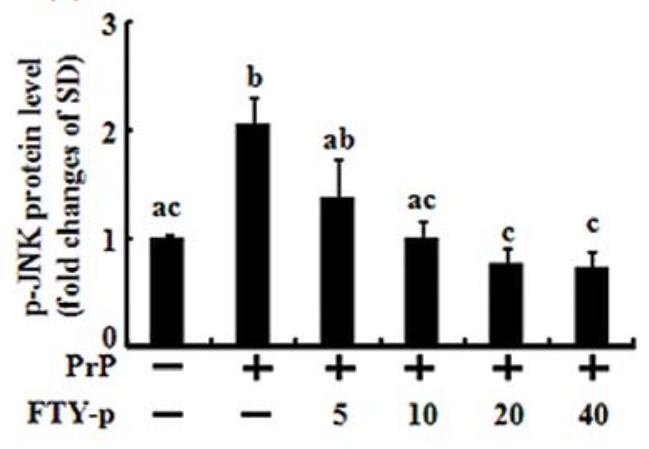

(C)
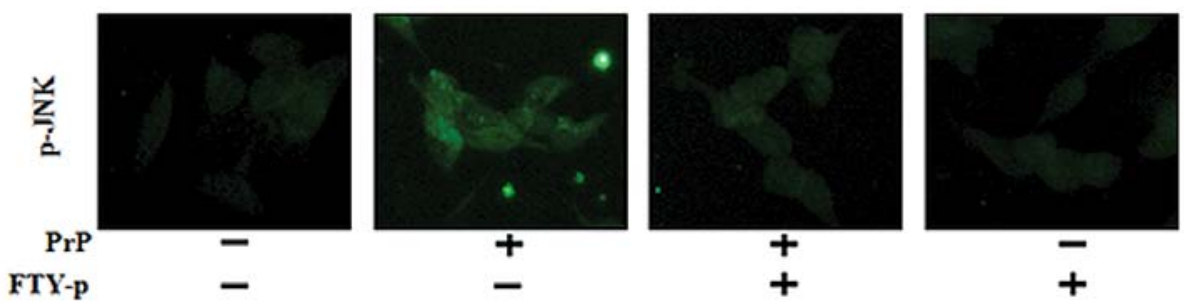

(D)
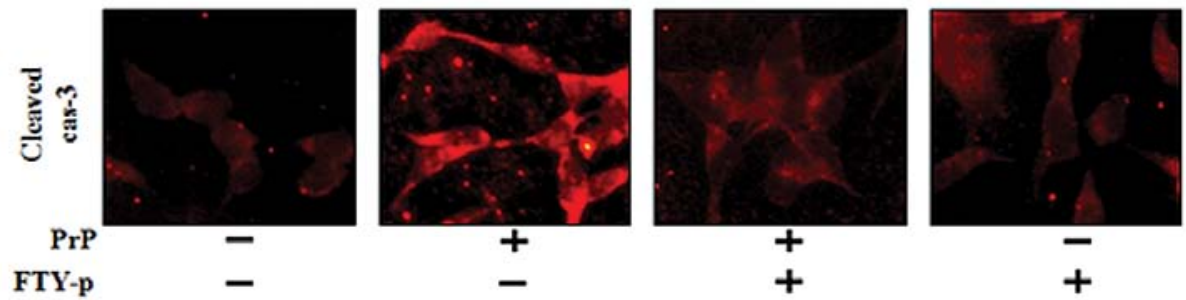

Figure 3. Effects of treatment with FTY720 phosphate (FTY-p) on c-jun N-terminal kinase (JNK) phosphorylation on PrP (106-126)-mediated neuronal apoptosis. (A) SH-SY5Y cells were pre-treated with various doses of FTY-p (5, 10, 20 and $40 \mu \mathrm{M}$ ) for $1 \mathrm{~h}$ and then exposed to 50 $\mu \mathrm{M}$ PrP (106-126) for $24 \mathrm{~h}$. The treated cells were assessed for JNK phosphorylation and cleavage of caspase-3 by western blot analysis. The results were normalized to $\beta$-actin. (B) Levels of p-JNK were normalized to $\beta$-actin. The experiments were repeatedly performed to confirm the results. Mean values denoted by a common alphabetical symbol do not differ significantly. Bars labeled with different letters indicate significant differences among each group of bars according to Duncan's test ( $<<0.05)(\mathrm{C}$ and D) Cells were pre-treated with $10 \mu \mathrm{M}$ of FTY-p $(1 \mathrm{~h})$ and then exposed to $50 \mu \mathrm{M}$ of $\operatorname{PrP}(106-126)$ for $24 \mathrm{~h}$. The treated cells were immunostained with p-JNK (green, C) and cleaved caspase-3 antibodies (red, D) and observed under a fluorescence microscope. Cas-3, caspase-3.

the release of cytochrome $c$, as did treatment with FTY720-p, which inhibited PrP (106-126)-mediated mitochondrial dysfunction (Fig. 4E). These results suggest that FTY720-p prevents PrP (106-126)-induced mitochondrial dysfunction and apoptosis by regulating the activation of JNK.

\section{Discussion}

FTY720 has a variety of neuroprotective effects on the CNS, protecting the CNS against MS, injury, as well as cerebral ischemia $(15,28)$. However, to our knowledge, the effects of FTY720 on neurodegenerative diseases, particularly prionmediated diseases, have not yet been reported. In this study, we demonstrate that FTY720 acts as a protective regulator of neuronal cell damage induced by PrP (106-126) and that FTY720 mediates the activation of JNK. Treatment with $\operatorname{PrP}$ (106-126) effectively inhibited cell survival by inducing mitochondrial dysfunction. However, treatment with FTY720-p blocked PrP (106-126)-mediated mitochondrial damage and apoptosis by inactivating JNK. Similarly, the abrogation of JNK led to the recovery of the impaired mitochondrial function and decreased cell viability induced by $\operatorname{PrP}(106-126)$.
FTY720, a non-selective S1P receptor agonist that induces sustained lymphopenia and accumulates in the CNS, represents a novel treatment modality for MS (29). In 2010, it became the first oral drug to be approved by the Food and Drug Administration for clinical use in the treatment of MS. In addition to its use in the treatment of MS, FTY720 exerts pleiotropic effects on oligodendrocytes and other neuronal cells (30). In a previous study, treatment of rodent-derived oligodendrocyte progenitor cells with FTY720 $(1 \mu \mathrm{M})$ rescued them from death induced by growth factor withdrawal, treatment with cytokines and exposure to activated microglia conditioned medium via extracellular signal-regulated kinase $1 / 2$ and Akt signaling (18). In accordance with these studies, in this study, we found that FTY720 exerts neuroprotective effects against prion diseases. FTY720 hindered neuronal cell death induced by $\operatorname{PrP}$ (106-126) by inactivating JNK (Figs. 1 and 3).

The association between mitochondrial function and neurodegenerative diseases has been investigated to a certain extent. The mitochondria are critical regulators of cell death and a key feature of neurodegeneration (31). Mitochondrial dysfunction occurs at an early stage and is involved in disease pathogenesis (31). The regulation of mitochondrial homeostasis affects the progression of neurodegenerative diseases, 
(A)

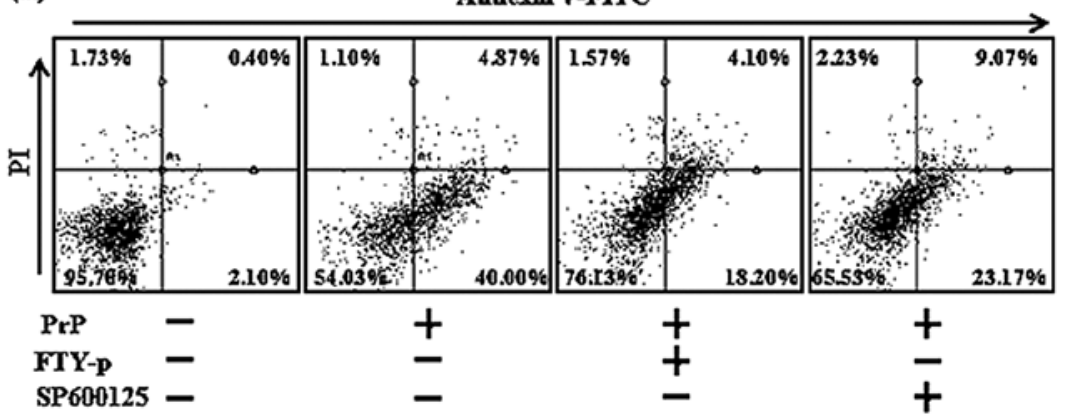

(B)

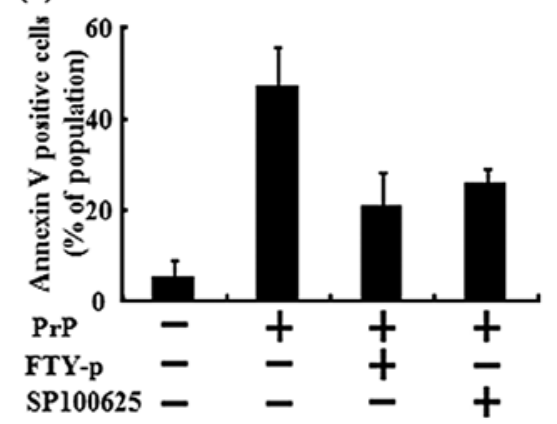

(C)
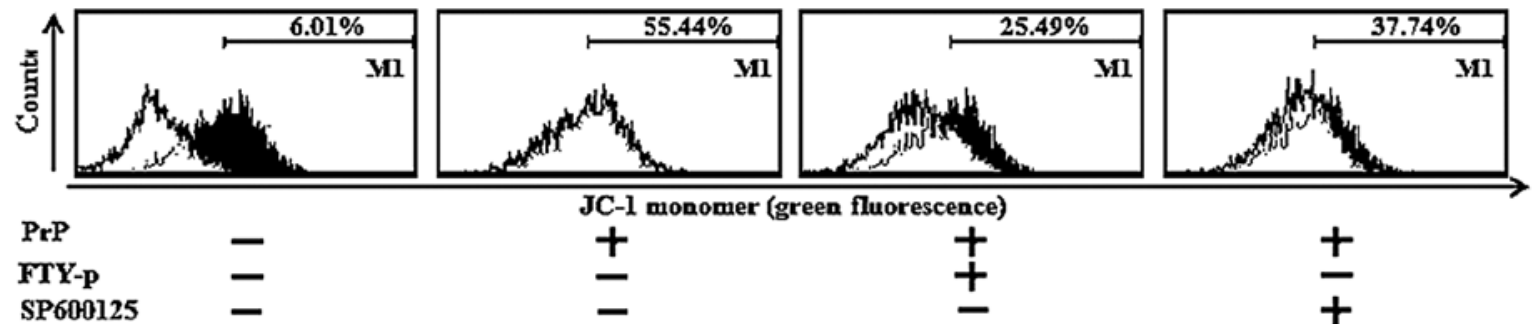

(D)

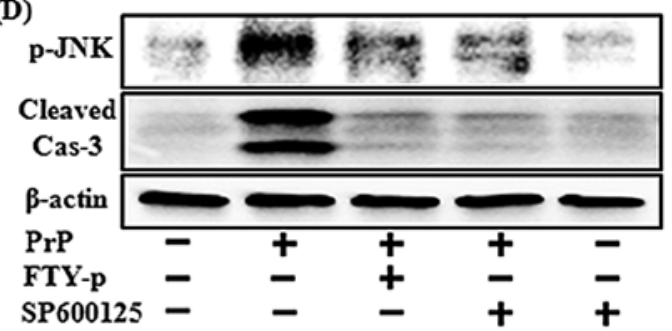

JC-1 monomer (green fluorescence)
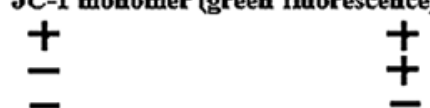

(E) $\frac{ \pm}{+}$

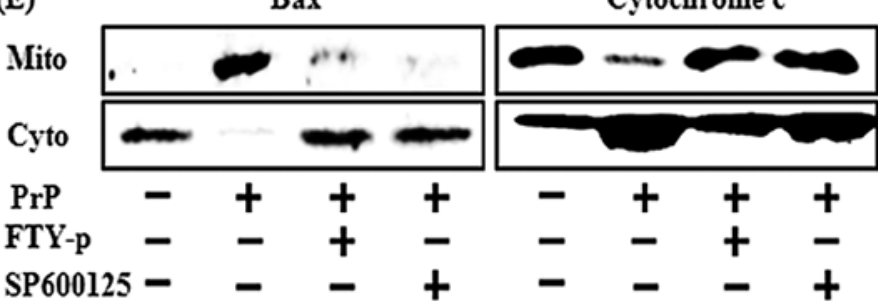

Figure 4. FTY720 phosphate (FTY-p) decreases PrP (106-126)-induced neurotoxicity by regulating c-jun N-terminal kinase (JNK) signaling in neuronal cells. (A) SH-SY5Y cells were pre-treated with $10 \mu \mathrm{M}$ of FTY720-p or JNK inhibitor ( $2 \mu \mathrm{M}$ of SP600125) for $1 \mathrm{~h}$ and then exposed to 50 $\mu \mathrm{M}$ PrP (106-126) for $24 \mathrm{~h}$. Cell viability was measured by Annexin V assay. (B) Bar graph indicates the averages of Annexin V-positive cells. Data were analyzed using analysis of variance (ANOVA) and the Duncan multiple range test $(\mathrm{p}<0.05)$. Bars depict the means $\pm \mathrm{SD}(\mathrm{n}=3)$. The experiments were repeatedly performed to confirm the results. Data were analyzed using analysis of variance (ANOVA) and the Duncan multiple range test ( $<<0.05$ ). Mean values denoted by a common alphabetical symbol do not differ significantly. Bars labeled with different letters indicate significant differences among each group of bars according to Duncan's test (p<0.05). (C) SH-SY5Y cells were treated as described in (A) and those which presented JC-1 in a mono form (green) were examined by flow cytometry. M1 represents the population of JC-1 monomeric cells. (D) SH-SY5Y cells were treated as described in (A) and the cells were assessed for JNK phosphorylation and caspase-3 cleavage by western blot analysis. Results were normalized to $\beta$-actin. (E) SH-SY5Y cells were treated as described in (A) and the cells were homogenized in mitochondrial buffer. The separation of cytosol and mitochondrial extracts was analyzed by western blot analysis using antibodies against cytochrome $c$ and Bax protein. Cas-3, caspase-3.

including Alzheimer's and Parkinson's diseases (32). The typical pattern of neurotoxicity in prion diseases is due to mitochondrial damage (13). Consistent with these studies, in our study, FTY720 inhibited prion-mediated mitochondrial disruption and neurotoxicity. Apoptosis was induced in PrP (106-126)-treated cells, which decreased MTP values and induced the translocation of Bax protein to the mitochondria (Fig. 2). However, treatment with FTY720-p attenuated the damaging effects induced by the prion peptide in neuronal cells (Fig. 2).

$\mathrm{JNK}$ is considered a competent inducer of the release of cy tochrome $c$ from the intermembrane space of the brain mitochondria and of the translocation of Bax to the mitochondria, thus initiating an essential step in mitochondrion-dependent apoptosis $(27,33)$. To the best of our knowledge, the effects of FTY720 on JNK signaling in neurodegenerative disorders have not been reported to date. In the present study, we demonstrate that FTY720 prevents PrP (106-126)-mediated neuronal cell mitochondrial disruption by inactivating JNK (Figs. 3 and 4).

It remains to be clarified which S1P receptors are related to the neuroprotective effects of FTY720. Further studies are required to determine the influence of S1P receptors on PrP (106-126)-mediated neurotoxicity in vitro and/or in vivo. As the Food and Drug Administration has already approved the oral drug that readily crosses the blood-brain barrier, FTY720 is considered an attractive candidate for neuroprotection. To the best of our knowledge the results of the current study, for the first time, attest to its ability to promote the survival of neuronal cells and protect them against prion-mediated neuronal damage by protecting the mitochondria. Future studies should include an evaluation of the therapeutic effects of FTY720 in conjunction with prion disease in in vivo animal models, as well as an examination of the hypothesis that prion-related neurodegenerative diseases may be attenuated by treatment with FTY720. 


\section{Acknowledgements}

This study was supported by the National Research Foundation of Korea Grant funded by the Korean Government (2013R1A2A2A01009614).

\section{References}

1. Prusiner SB: Prions. Proc Natl Acad Sci USA 95: 13363-13383, 1998.

2. Seo JS, Moon MH, Jeong JK, et al: SIRT1, a histone deacetylase, regulates prion protein-induced neuronal cell death. Neurobiol Aging 33: 1110-1120, 2012.

3. Seo JS, Seol JW, Moon MH, Jeong JK, Lee YJ and Park SY: Hypoxia protects neuronal cells from human prion protein fragment-induced apoptosis. J Neurochem 112: 715-722, 2010.

4. Watt NT, Taylor DR, Gillott A, Thomas DA, Perera WSS and Hooper NM: Reactive oxygen species-mediated beta-cleavage of the prion protein in the cellular response to oxidative stress. J Biol Chem 280: 35914-35921, 2005.

5. Jeong JK, Seo JS, Moon MH, Lee YJ, Seol JW and Park SY: Hypoxia-inducible factor-1 $\alpha$ regulates prion protein expression to protect against neuron cell damage. Neurobiol Aging 33: 1006.e1-10, 2012.

6. Gray F, Chrétien F, Adle-Biassette H, et al: Neuronal apoptosis in Creutzfeldt-Jakob disease. J Neuropathol Exp Neurol 58: 321-328, 1999.

7. Liberski PP, Sikorska B, Bratosiewicz-Wasik J, Gajdusek DC and Brown P: Neuronal cell death in transmissible spongiform encephalopathies (prion diseases) revisited: from apoptosis to autophagy. Int J Biochem Cell Biol 36: 2473-2490, 2004.

8. Forloni G, Angeretti N, Chiesa R, et al: Neurotoxicity of a prion protein fragment. Nature 362: 543-546, 1993.

9. Brown DR, Schmidt B and Kretzschmar HA: Role of microglia and host prion protein in neurotoxicity of a prion protein fragment. Nature 380: 345-347, 1996.

10. Florio T, Thellung S, Amico C, et al: Prion protein fragment 106-126 induces apoptotic cell death and impairment of L-type voltage-sensitive calcium channel activity in the GH3 cell line. J Neurosc Res 54: 341-352, 1998.

11. Ettaiche M, Pichot R, Vincent J-P and Chabry J: In vivo cytotoxicity of the prion protein fragment 106-126. J Biol Chem 275: 36487-36490, 2000.

12. Jeong JK, Moon MH, Lee YJ, Seol JW and Park SY: Autophagy induced by the class III histone deacetylase Sirt 1 prevents prion peptide neurotoxicity. Neurobiol Aging 34: 146-156, 2013.

13. O'Donovan CN, Tobin D and Cotter TG: Prion protein fragment PrP-(106-126) induces apoptosis via mitochondrial disruption in human neuronal SH-SY Y Y cells. J Biol Chem 276: 43516-43523, 2001.

14. Kiuchi M, Adachi K, Kohara T, et al: Synthesis and immunosuppressive activity of 2-substituted 2-aminopropane-1,3-diols and 2-aminoethanols. J Med Chem 43: 2946-2961, 2000.

15. Wei Y, Yemisci M, Kim HH, et al: Fingolimod provides long-term protection in rodent models of cerebral ischemia. Ann Neurol 69: 119-129, 2011.

16. Kihara A and Igarashi Y: Production and release of sphingosine 1-phosphate and the phosphorylated form of the immunomodulator FTY720. Biochim Biophys Acta 1781: 496-502, 2008.
17. Stessin AM, Gursel DB, Schwartz A, et al: FTY720, sphingosine 1-phosphate receptor modulator, selectively radioprotects hippocampal neural stem cells. Neurosci Lett 516: 253-258, 2012.

18. Miron VE, Schubart A and Antel JP: Central nervous system-directed effects of FTY720 (fingolimod). J Neurol Sci 274: 13-17, 2008

19. Harada J, Foley M, Moskowitz MA and Waeber C: Sphingosine-1-phosphate induces proliferation and morphological changes of neural progenitor cells. J Neurochem 88: 1026-1039, 2004

20. Chun J, Weiner JA, Fukushima N, et al: Neurobiology of receptor-mediated lysophospholipid signaling. From the first lysophospholipid receptor to roles in nervous system function and development. Ann N Y Acad Sci 905: 110-117, 2000.

21. Edsall LC, Pirianov GG and Spiegel S: Involvement of sphingosine 1-phosphate in nerve growth factor-mediated neuronal survival and differentiation. J Neurosci 17: 6952-6960, 1997.

22. Jackson SJ, Giovannoni G and Baker D: Fingolimod modulates microglial activation to augment markers of remyelination. J Neuroinflammation 8: 76, 2011.

23. Deogracias R, Klein C, Matsumoto T, et al: Expression of brain-derived neurotrophic factor is regulated by fingolimod (FTY720) in cultured neurons. Mult Scler 14: S243, 2008.

24. Brinkmann V, Cyster JG and Hla T: FTY720: sphingosine 1-phosphate receptor-1 in the control of lymphocyte egress and endothelial barrier function. Am J Transplant 4: 1019-1025, 2004.

25. Valentine WJ,Kiss GN,Liu J,et al: (S)-FTY720-vinylphosphonate, an analogue of the immunosuppressive agent FTY720, is a pan-antagonist of sphingosine 1-phosphate GPCR signaling and inhibits autotaxin activity. Cell Signal 22: 1543-1553, 2010.

26. Tournier C, Hess P, Yang DD, et al: Requirement of JNK for stress-induced activation of the cytochrome c-mediated death pathway. Science 288: 870-874, 2000.

27. Tsuruta F, Sunayama J, Mori Y, et al: JNK promotes Bax translocation to mitochondria through phosphorylation of 14-3-3 proteins. EMBO J 23: 1889-1899, 2004.

28. Ehling R, Berger T and Reindl M: Multiple sclerosis - established and novel therapeutic approaches. Cent Nerv Syst Agents Med Chem 10: 3-15, 2010.

29. Gonzalez-Cabrera PJ, Cahalan SM, Nguyen N, et al: S1P(1) receptor modulation with cyclical recovery from lymphopenia ameliorates mouse model of multiple sclerosis. Mol Pharmacol 81: 166-174, 2012.

30. Kim HJ, Miron VE, Dukala D, et al: Neurobiological effects of sphingosine 1-phosphate receptor modulation in the cuprizone model. FASEB J 25: 1509-1518, 2011.

31. Lin MT and Beal MF: Mitochondrial dysfunction and oxidative stress in neurodegenerative diseases. Nature 443: 787-795, 2006.

32. Jeong JK, Moon MH, Lee YJ, Seol JW and Park SY: Melatonin-induced autophagy protects against human prion protein-mediated neurotoxicity. J Pineal Res 53: 138-146, 2012.

33. Schroeter H, Boyd CS, Ahmed R, et al: c-Jun N-terminal kinase (JNK)-mediated modulation of brain mitochondria function: new target proteins for JNK signalling in mitochondrion-dependent apoptosis. Biochem J 372: 359-369, 2003. 